\title{
Magnetotransport through Nanoconstriction in Ferromagnetic Semiconductor (Ga,Mn)As
}

\author{
T. Figielski ${ }^{a}$, T. Wosiński ${ }^{a}$, O. Pelya ${ }^{a}$, J. SAdowski ${ }^{a, b}$, \\ A. MoraWski ${ }^{a}, \mathrm{~A} . \mathrm{MAzKOSA}^{a}, \mathrm{~W} . \mathrm{Dobrowolski}^{a}$, \\ J. JAGIELSKI ${ }^{c}$ AND J. WRÓBEL ${ }^{a}$ \\ ${ }^{a}$ Institute of Physics, Polish Academy of Sciences \\ al. Lotników 32/46, 02-668 Warszawa, Poland \\ ${ }^{b}$ Max-lab, Lund University, 22100 Lund, Sweden \\ 'Institute of Electronic Materials Technology, 01-919 Warszawa, Poland \\ We studied narrow (submicron) constrictions in the layers of ferro- \\ magnetic semiconductor (Ga, Mn)As. We have demonstrated a contribution \\ of the quantum localization effects to the magnetoresistance of the con- \\ stricted samples. We have also found a negative contribution of a domain \\ wall trapped in the constriction to the resistance, due presumably to the \\ erasing of the localization effects by the domain wall.
}

PACS numbers: 73.63.-b, 75.60.-d, 73.20.Fz

\section{Introduction}

The object of this study is a ferromagnetic semiconductor $(\mathrm{Ga}, \mathrm{Mn}) \mathrm{As}$, in which a few percent of Ga atoms has been replaced by Mn atoms. Recent advance in the growth of ferromagnetic semiconductors based on III-V compounds brought about a unique possibility for the integrating electronic and magnetoelectronic devices, which might create a basis for future spin electronics $[1,2]$.

Our interest is focused on the phenomena, which occur in a narrow constriction fabricated from this material (Fig. 1a), particularly, on the charge transport through a magnetic domain wall (DW) trapped in the constriction. That subject has just emerged as a hot topic in the physics of ferromagnetic nanostructures $[3,4]$. 


\section{Samples}

In our study we used the wafers, which consisted of 50 nm-thick layers of $\mathrm{Ga}_{0.99} \mathrm{Mn}_{0.01} \mathrm{As}$, grown by the low-temperature molecular-beam epitaxy (LT-MBE) on insulating $\mathrm{GaAs}(001)$ substrates [5]. The Curie temperature, $T_{\mathrm{C}}$, in these layers was $17 \mathrm{~K}$. The layers exhibit metallic p-type conductivity. Individual samples of an area $(1 \times 3) \mathrm{mm}^{2}$ were separated in the grown layer by etching of a $20 \mu \mathrm{m}$-wide groove around them. We measured the sample conductance, $G$, as a function of a magnetic field, $B$, using the standard lock-in technique.

The reference non-constricted samples exhibit at low temperatures a large positive magnetoconductance (MC) extending far outside the field range of ferromagnetic hysteresis loop. This MC has been basically understood as the reduction of spin-dependent carrier scattering due to the aligning of localized Mn spins by an applied field. For a magnetic field perpendicular to the layer plane, additionally, a small negative contribution to the MC appears in a narrow range around $B=0$. This feature has been usually attributed to the rotation of Mn spins from its original in-plane direction to the perpendicular direction. However, as far as we know, no quantitative explanation of the MC in this material has been proposed, except for temperatures close to $T_{\mathrm{C}}[1,2]$.

(a)
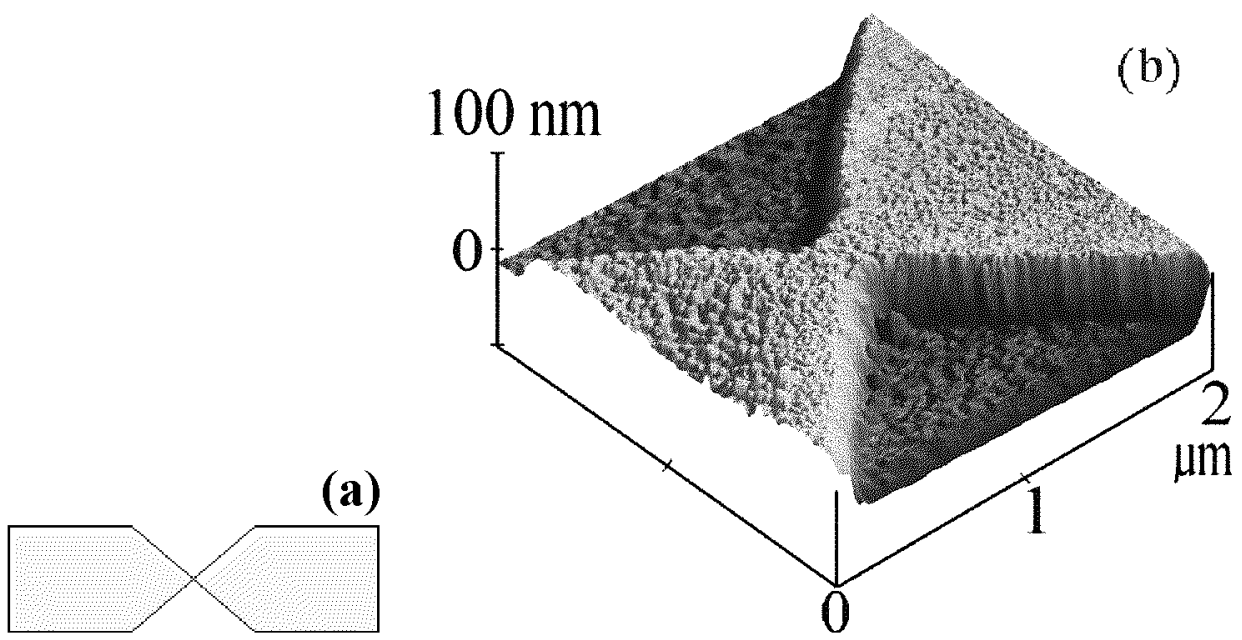

Fig. 1. Geometry of the constricted sample of ferromagnetic layer (a); atomicforce-microscope image of the constriction created by chemical etching (b).

Beginning the work, we first fabricated narrow constrictions in the ferromagnetic layers using electron-beam lithography and chemical etching; Fig. 1b. Unexpectedly, the samples with submicron constrictions obtained in this way were non-conducting at liquid helium temperatures, although their conductivity could be induced by illumination [6]. Presumably, the quenching of sample conductance 
is connected with an extra surface area denuded by the etching, which either enables penetration of oxygen into the constricted channel or generates a high density of trapping surface states.

Since the chemical etching turned out to be useless for the tailoring of nanoconstrictions, we next applied for this aim an ion implantation method. We found that implantation of oxygen ions with energy of $25 \mathrm{keV}$ and a dose of $5 \times 10^{13}$ ions $/ \mathrm{cm}^{2}$ caused a suppression of both the conductivity and ferromagnetism in the layer [6]. We implanted oxygen ions into the layer through a mask, which consisted of a thick resist deposited on the top of the layer and contained windows patterned by electron-beam lithography. The lithographic widths of the constrictions were in a range of $0.2-0.6 \mu \mathrm{m}$.

\section{Magnetoconductance}

After tailoring, the MR of the constricted samples displays new interesting features, which are clearly seen in Fig. 2, where the sample magnetoconductance is plotted as a function of a magnetic field applied perpendicular to the layer plane.

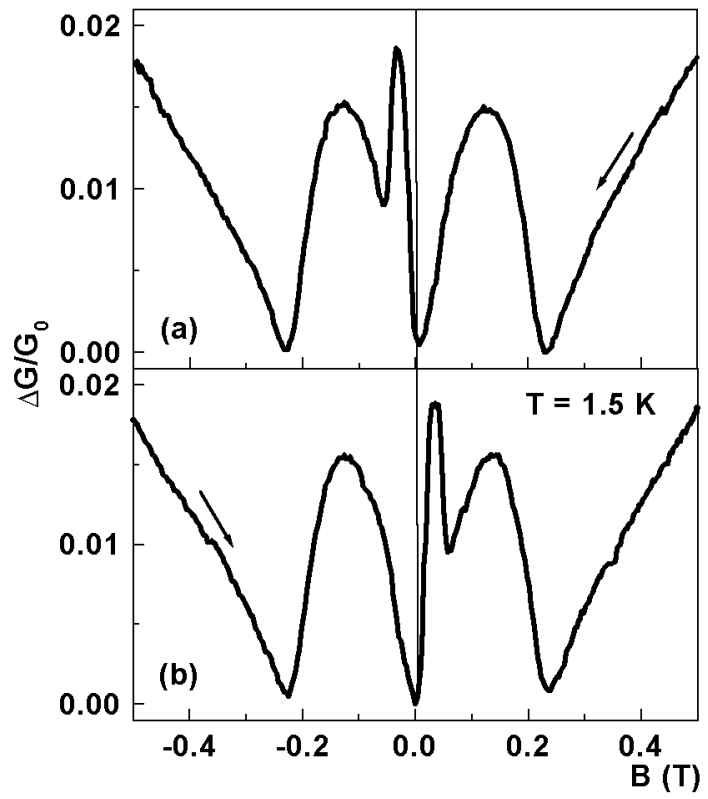

Fig. 2. Magnetoconductance, $[\mathrm{G}(\mathrm{B})-\mathrm{G}(0)] / \mathrm{G}(0)$, of the constricted sample of $\mathrm{Ga}_{0.99} \mathrm{Mn}_{0.01} \mathrm{As}$, created by oxygen ion implantation, as a function of decreasing (a) and increasing (b) magnetic field applied perpendicular to the layer plane, at a temperature of $1.5 \mathrm{~K}$. 
Going from strong (positive) to weak field one meets a crossover from the positive to the negative MC, which has been also observed in reference non-constricted samples. But now, as the field is reduced further, the positive MC appears again and, moreover, after crossing the point $B=0$ (i.e. after reversal of the magnetic field direction) a sharp peak of an enhanced conductance appears.

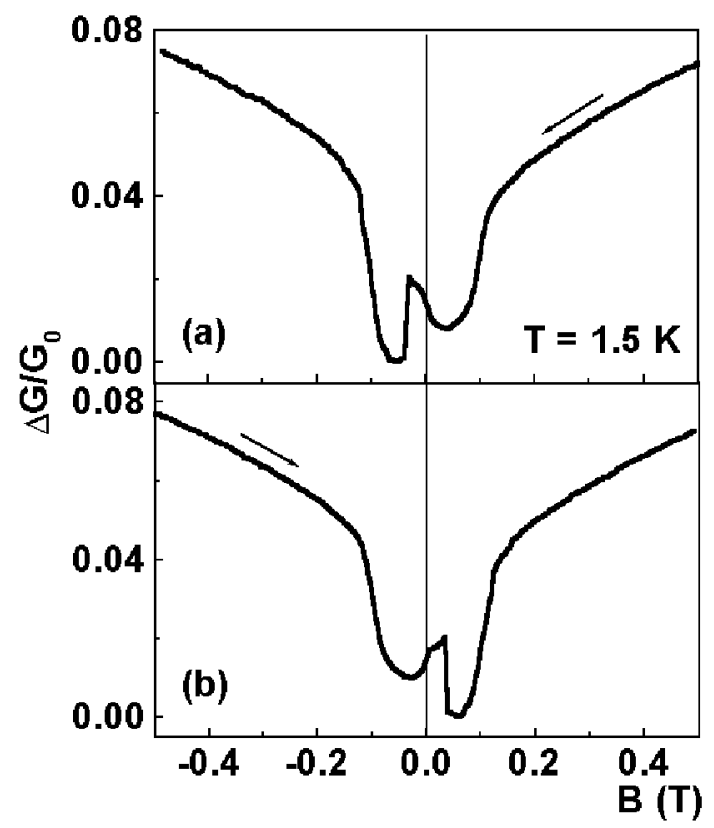

Fig. 3. The same as in Fig. 2 but for a magnetic field applied parallel to the layer plane.

Analogical dependence of the MC is observed if to start from the opposite direction of $B$. Positions of the peaks of enhanced conductance reflect the hysteresis loop and coincide with the coercive field, $\left|H_{c}\right|=300$ Oe. Similar features have been also observed in a weak magnetic field parallel to the layer plane; Fig. 3.

\section{Effects of domain wall}

To understand these results we must keep in mind that a magnetic domain wall tends to localize itself near the constriction, in order to minimize its energy. Moreover, such a geometrically constrained wall differs from that in a bulk ferromagnet (it is neither Bloch nor Néel wall) [7].

Several authors, cf. [8], studied theoretically the effect of electron scattering at domain walls on the resistivity of ferromagnetic metals. Theories predict generally a large positive contribution of DW to the resistivity if the wall is thin enough 
compared to the Fermi wavelength, and if the charge transport through the wall is ballistic.

On the contrary, in our constricted samples we observe a distinct increase in the conductivity at the $H_{\mathrm{c}}$, i.e. when a domain wall is nucleated in the constriction. This clearly shows a negative contribution of DW to the resistivity. This is, however, not very surprising since several authors have also identified a negative contribution of domain walls to the resistivity in metallic ferromagnetic microwires [9].

In turn, Tatara and Fukuyama [10] predicted that a DW destroys the electron phase coherence necessary for weak localization in the case of a dirty metal, leading to a negative contribution of DW to the resistivity. Basically, this predicted effect is due to misalignment of the localized spins inside the wall. We believe that our results are just manifestation of such an effect.

\section{Quantum localization}

The ferromagnetic semiconductor studied by us differs from typical ferromagnetic metals by a high disorder and falls into the category of dirty metals. In fact, the product $k_{\mathrm{F}} l$ ( $k_{\mathrm{F}}$ is the Fermi wave number and $l$ is the hole mean elastic free path) is of the order of unity at liquid helium temperatures. In that case the semiclassical picture of charge transport fails.

However, as shown by Minkov et al. [11], the conductivity may be then still treated as quasi-diffusive but with quantum corrections due mainly to the quantum interference. In the following we will discuss our results adopting the conventional picture of weak localization.

Weak localization (WL) arises due to the constructive interference between pairs of partial waves corresponding to an electron travelling diffusively along a closed trajectory in opposite directions. That interference enhances electron backscattering, and leads to a negative contribution to the conductivity. Magnetic field suppresses WL as it introduces a phase difference between the time-reversed paths, cf. [12].

It is worthwhile to pay attention here to a surprising general similarity between the field-dependent MC of ferromagnetic ( $\mathrm{Ga}, \mathrm{Mn}$ ) As and the MC observed in non-magnetic metallic films that has been explained in terms of WL theory [12]. We will not discuss this issue here, instead, we propose that the positive MC appearing in the constricted samples around $B=0$ is a manifestation of the localization in a narrow quasi-1D channel. It is actually possible to fit our MC data to the conventional WL theory [13] assuming reasonable material parameters, which will be discussed elsewhere.

Our principal observation is that a sharp conductance peak appears in a magnetic field equal to the coercive field, i.e. just when a domain wall is nucleated in the constriction. This can be understood as due to the erasing of quantum local- 
ization by a DW in the constriction, just as predicted by Tatara and Fukuyama [10] and other authors $[14,15]$. This is the main conclusion of this work.

\section{Final remark}

We still want to pay attention to an interesting mechanism of the dephasing due to a DW trapped in nanoconstriction. If the width of a DW is comparable to the constriction width, the rotating magnetization direction can be inhomogeneous in two dimensions instead of one as for a long DW. For instance, we could imagine ourselves that a DW has a mixed Bloch-Néel character, where the relative contribution of each component changes across the constriction width.

Then, if the spin of a travelling particle follows adiabatically the direction of local magnetic field, $\boldsymbol{B}_{i}$, inside a DW, and the particle makes a closed loop to return to its starting point, its wave function acquires an additional geometrical Berry-phase [14]. A measure of this phase is the solid angle subtended by the vector $\boldsymbol{B}_{i}$ experienced by the travelling particle, multiplied by a factor depending on the spin value and the direction of circulation. So, when this solid angle is different from zero, the difference in the geometrical-phases between the time-reversed paths will tend to destroy the constructive interference and to erase the localization effects.

\section{Acknowledgments}

We are indebted to M. Arciszewska and E. Łusakowska for their assistance in the work. The work was partly supported within European Community program ICA1-CT-2000-70018 (Centre of Excellence CELDIS).

\section{References}

[1] F. Matsukura, H. Ohno, A. Shen, Y. Sugawara, Phys. Rev. B 57, R2037 (1998).

[2] A. Oiwa, S. Katsumoto, A. Endo, M. Hirasawa, Y. Iye, H. Ohno, F. Matsukura, A. Shen, Y. Sugawara, Solid. State Commun. 103, 209 (1997).

[3] R. Danneau, P. Warin, J.P. Attane, I. Petej, C. Beigne, C. Fermon, O. Klein, A. Marty, F. Ott, Y. Samson, M. Viret, Phys. Rev. Lett. 88, 157201 (2002).

[4] N. García, G.G. Qiang, I.G. Saveliev, Appl. Phys. Lett. 80, 1785 (2002).

[5] J. Sadowski. J.Z. Domagała, J. Bąk-Misiuk, S. Koleśnik, M. Sawicki, K. Świątek, J. Kanski, L. Ilver, V. Strom, J. Vac. Sci. Technol. B 18, 1697 (2000).

[6] T. Figielski, T. Wosinski, A. Morawski, O. Pelya, J. Sadowski, A.L. Toth, J. Jagielski, Phys. Status Solidi A 195, 228 (2003).

[7] P. Bruno, Phys. Rev. Lett. 83, 2425 (1999).

[8] H.X. Tang, R.K. Kawakami, D.D. Awschalom, M.L. Roukes, Phys. Rev. Lett. 90, $107201(2003)$.

[9] K. Miyake, K. Shigeto, K. Mibu, T. Shinjo, T. Ono, J. Appl. Phys. 91, 3468 (2002). 
[10] G. Tatara, H. Fukuyama, Phys. Rev. Lett. 78, 3773 (1997).

[11] G.M. Minkov, O.E. Rut, A.V. Germanenko, A.A. Sherstobitov, B.N. Zvonkov, E.A. Uskova, A.A. Birukov, Phys. Rev. B 65, 235322 (2002).

[12] G. Bergmann, Phys. Rep. 107, 1 (1984).

[13] C.W.J. Beenakker, H. van Houten, Phys. Rev. B 38, 3232 (1988).

[14] Y. Lyanda-Geller, I.L. Aleiner, P.M. Goldbart, Phys. Rev. Lett. 81, 3215 (1998).

[15] Y. Takane, Y. Koyama, J. Phys. Soc. Jpn. 69, 328 (2000). 